\title{
The Hawking Temperature Intensive Crisis and a Possible Solution that Leads to an Intensive Schwarzschild Surface Temperature
}

\author{
Espen Gaarder Haug ${ }^{1}$
}

\author{
${ }^{1}$ Norwegian University of Life Sciences, Oslo, Norway \\ Correspondence: Espen Gaarder Haug, Norwegian University of Life Sciences, Oslo, Norway. E-mail: espenhaug@mac.com
}

Received: March 22, 2019

doi:10.5539/apr.v11n3p56
Accepted: April 15, $2019 \quad$ Online Published: May 6, 2019

URL: https://doi.org/10.5539/apr.v11n3p56

\begin{abstract}
:
Crothers and Robitaille have recently pointed out that the Hawking temperature and the Unruh temperature are not intensive and how this is inconsistent with thermodynamics, which suggests that the theory around the temperature of black holes is flawed, incomplete, or at least not fully understood. Here we offer a modified Newtonian type acceleration field linked to the Planck scale that leads to a new modified intensive Schwarzschild surface temperature for so-called black holes.
\end{abstract}

Keywords: Hawking temperature, Unruh temperature, Planck scale, Schwarzschild radius, black holes

\section{The Intensive Crisis in the Hawking Temperature and the Unruh Temperature}

In 1974, Hawking introduced the idea of black hole radiation and a corresponding temperature at the black hole's surface, better known today as Hawking radiation and Hawking temperature, see also Hawking (1976). The Hawking temperature is given by

$$
T_{H}=\frac{\hbar g}{2 \pi c k_{B}}
$$

where $c$ is the speed of light, $k_{b}$ is the Boltzmann constant, and $g$ is the gravitational acceleration field. The Hawking temperature was originally stated as what

"one would expect if the black hole was a body with temperature of $(\kappa / 2 \pi)\left(\hbar / 2 k_{b}\right) \ldots$.."

- Stephen Hawking, 1974

where $\kappa$ is the surface gravity of the black hole. And the Unruh temperature is very similar to the Hawking temperature

$$
T_{U}=\frac{\hbar a}{2 \pi c k_{B}}
$$

The Newton gravitational acceleration at the Schwarzschild radius is given by

$$
g=\frac{G M}{R_{s}^{2}}=\frac{G M}{\left(\frac{2 G M}{c^{2}}\right)^{2}}=\frac{c^{4}}{4 G M}
$$

and when replaced in the Hawking temperature it is

$$
T_{H}=\frac{\hbar c^{3}}{8 \pi G M k_{B}}
$$

When the mass increases, the Hawking temperature and Unruh temperature at the Schwarzschild radius will fall. It has been known for a long time that the Hawking temperature not is intensive, see, for example, Hochberg and Juan (1997). However, Robitaille (2018); Crothers and Robitaille $(2018,2019)$ are likely the first ones to point out clearly that this seems to be in strong conflict with thermodynamics. This possibly leads to an intensive crisis in the Hawking temperature and the Unruh temperature at the Schwarzschild radius, since temperature must, according to thermodynamics, be intensive, see Landsberg (1961). Landsberg even claimed that the intensity and extensive properties and their relations 
should be considered the fourth law of thermodynamics. So, could this indicate that the Hawking temperature is flawed or incomplete? In this paper, we examine the Hawking and Unruh intensive crisis.

We will assert that it is important to be careful about criticism of predictions at the Schwarzschild radius. The smallest Schwarzschild radius is the Planck length and, we will claim, the Schwarzschild radius is directly linked to the Planck scale. Recent research also shows that we can measure the Schwarzschild radius without any knowledge of general relativity theory or Newton's gravitational constant. The Schwarzschild radius is, in our view, essential for gravity, but what does it truly represent? See Haug (2019). Yet, the importance of the Schwarzschild radius seems to have little to do with the traditional view on black holes; something we will get back to later. It is also worth mentioning that a radius equivalent to the so-called Schwarzschild radius was pointed out by Michell (1784), based on Newtonian theory alone.

At the Planck scale, several physical laws could break down, including Lorentz symmetry; this is also predicted by several quantum gravity theories (Reyes, Ossandon, \& Reyes, 2005; Hees et al., 2017), so what we normally consider "laws" do not necessary hold at the Planck scale. The Planck scale is very special indeed. However, we do not expect the requirement of intensity of temperature to be one of the rules that breaks down at the Planck scale, and we think that the Crothers and Robitaille paper, which points out the intensive crisis in the Hawking and Unruh temperatures, is valid here. This means the intensive crisis should be investigated further. After all, there is not a unified quantum gravity theory yet; something is missing.

\section{Modified Gravitational Acceleration Field}

The views and analysis in this section were first suggested by Haug in early 2018, before Crothers and Robitaille pointed out the intensive crisis in the Hawking and Unruh temperatures. Thus, there is an interesting timeline with regard to thoughts on this subject in very recent history; we did not try to come up with the modification in gravitational acceleration field (as suggested below) simply to tackle the intensive crisis in Hawking and Unruh temperatures. In fact, we have been working on other gravity topics and came up with this suggestion before we had heard of the intensive crisis. The modified gravitational acceleration field, even if somewhat ad-hoc, is based on deep tinkering with gravity at the Planck scale and we have contributed to the literature on this subject previously.

The gravitational acceleration field is unrealistically low under classical Newtonian (Newton (1686)) physics at the Schwarzschild radius. And yet the escape velocity at the Schwarzschild radius is always the speed of light, as we would expect. Assume a super-massive object that is $10^{14}$ solar masses. The gravitational acceleration field at the Schwarzschild radius is, under Newton's universal gravitation, only

$$
g=\frac{G M}{R^{2}}=\frac{G M}{\left(2 \frac{G M}{c^{2}}\right)^{2}} \approx 0.152 \mathrm{~m} / \mathrm{s}^{2}
$$

How can the escape velocity be $c$ and at the same time the surface gravity field is much weaker than that on Earth, where it is about $9.8 \mathrm{~m} / \mathrm{s}^{2}$ ? Further, we will suggest that no acceleration field can be stronger than the Planck acceleration field

$$
a_{p}=\frac{c^{2}}{l_{p}} \approx 5.56092 \times 10^{51} \mathrm{~m} / \mathrm{s}^{2}
$$

If the shortest possible time interval during which something can undergo acceleration is one Planck second, then if an object undergoes Planck acceleration for this time interval, it will reach the speed of light

$$
a_{p} t_{p}=\frac{c^{2}}{l_{p}} \frac{l_{p}}{c}=c
$$

As matter (with mass) cannot travel at the speed of light, in our interpretation this means that only a Planck mass particle can undergo this acceleration. As shown by Haug in a series of papers, the Planck particle is likely at absolute rest and within one Planck second will dissolve into pure energy, see Haug (2018e). This also explains why the mass can accelerate from rest-mass to the speed of $c$ within a Planck second; it has to dissolve into pure energy in this time frame. That is, the Planck mass particle dissolves into massless particles, photons, within one Planck second. From mathematical atomism Haug $(2014$, 2016b), only the Planck mass particle can do this within a Planck second. We will assume the Planck acceleration is what we have at the Schwarzschild radius. Further, we will assume the inverse square rule basically holds for a radius going out from the Schwarzschild radius rather than from the very center of the mass. Based on this scenario, our modified formula for gravitational acceleration field is 


$$
\begin{aligned}
g & \approx \frac{G M}{r^{2}-\left(\frac{2 G M}{c^{2}}\right)^{2}+\left(\frac{G M}{c^{2}}\right) l_{p}} \\
g & \approx \frac{c^{2} N l_{p}}{r^{2}-\left(2 N l_{p}\right)^{2}+N l_{p}^{2}}
\end{aligned}
$$

where $N$ is the number of Planck masses in the object. Now the acceleration field for a $10^{14}$ solar mass object at the Schwarzschild radius, $r=\frac{2 G M}{c^{2}}$, gives

$$
g \approx \frac{G M}{r^{2}-\left(\frac{2 G M}{c^{2}}\right)^{2}+\left(\frac{G M}{c^{2}}\right) l_{p}}=\frac{G M}{\left(\frac{2 G M}{c^{2}}\right)^{2}-\left(\frac{2 G M}{c^{2}}\right)^{2}+\left(\frac{G M}{c^{2}}\right) l_{p}}=\frac{c^{2}}{l_{p}} \approx 5.56092 \times 10^{51} \mathrm{~m} / \mathrm{s}^{2}
$$

Next, the mass of the Earth is approximately $2.74388 \times 10^{32}$ Planck masses. Further, the radius of the Earth is $6,371,000$ meters; this gives a gravitational acceleration field at the surface of Earth equal to

$$
g \approx \frac{c^{2} N l_{p}}{r^{2}+N l_{p}^{2}(1-N)}=\frac{c^{2} \times 2.74388 \times 10^{32} \times l_{p}}{6371000^{2}+\left(2 \times 2.74388 \times 10^{32} \times l_{p}\right)^{2}-2.74388 \times 10^{32} \times l_{p}} \approx 9.8194 \mathrm{~m} / \mathrm{s}^{2}
$$

Still, this formula always gives the Planck acceleration at the modified Schwarzschild radius. We have not evaluated this adjustment completely yet, and it should be investigated further.

\section{Back to the Intensive Crisis in Hawking Temperature}

As shown in the last section, our modified gravitational acceleration field is always the Planck acceleration at the Schwarzschild radius

$$
g_{r_{s}} \approx \frac{G M}{r_{s}^{2}-\left(\frac{2 G M}{c^{2}}\right)^{2}+\left(\frac{G M}{c^{2}}\right) l_{p}}=\frac{G M}{\left(\frac{2 G M}{c^{2}}\right)^{2}-\left(\frac{2 G M}{c^{2}}\right)^{2}+\left(\frac{G M}{c^{2}}\right) l_{p}}=\frac{c^{2}}{l_{p}} \approx 5.56092 \times 10^{51} \mathrm{~m} / \mathrm{s}^{2}
$$

which always gives a black hole surface temperature of

$$
T_{H}=\frac{h g_{r_{s}}}{4 \pi^{2} c k_{B}}=\frac{h c^{2}}{4 \pi^{2} c k_{B}}=\frac{\hbar}{l_{p} k_{B}} c \frac{1}{2 \pi}
$$

That is now intensive; no matter what the mass of the so-called black hole may be, our surface temperature is always the same. It is the Planck temperature divided by $2 \pi$, and it is intensive. This modified Schwarzschild surface temperature only corresponds to the Hawking temperature when we have a single Planck mass. The larger the black hole, the smaller is the Hawking temperature, while our temperature always stays the same. In our view, our temperature seems more logical and returns to the issue that there is a lack of quantization in general relativity and Newtonian theory.

Newton's gravitational constant is, in modern physics, only an observed constant used to calibrate gravity, so it fits observations. The gravitational constant was first indirectly found by Cavendish in 1798, when he was using a Cavendish apparatus to weigh the Earth (Cavendish, 1798). However, as Haug (2016a,c, 2018d) has pointed out, Newton's gravitational constant is very likely a composite constant of the form $G=\frac{l_{p}^{2} c^{3}}{\hbar}$. In 2014, McCulloch (2014) derived basically the same gravitational constant from Heisenberg's uncertainty principle; his formula was

$$
G=\frac{\hbar c}{m_{p}^{2}}
$$

and because the Planck mass can be written as

$$
m_{p}=\frac{\hbar}{l_{p}} \frac{1}{c}
$$

we can see that this is the same as the Haug formula 


$$
G=\frac{\hbar c}{\frac{\hbar}{l_{p}} \frac{1}{c} \frac{\hbar}{l_{p}} \frac{1}{c}}=\frac{l_{p}^{2} c^{3}}{\hbar}
$$

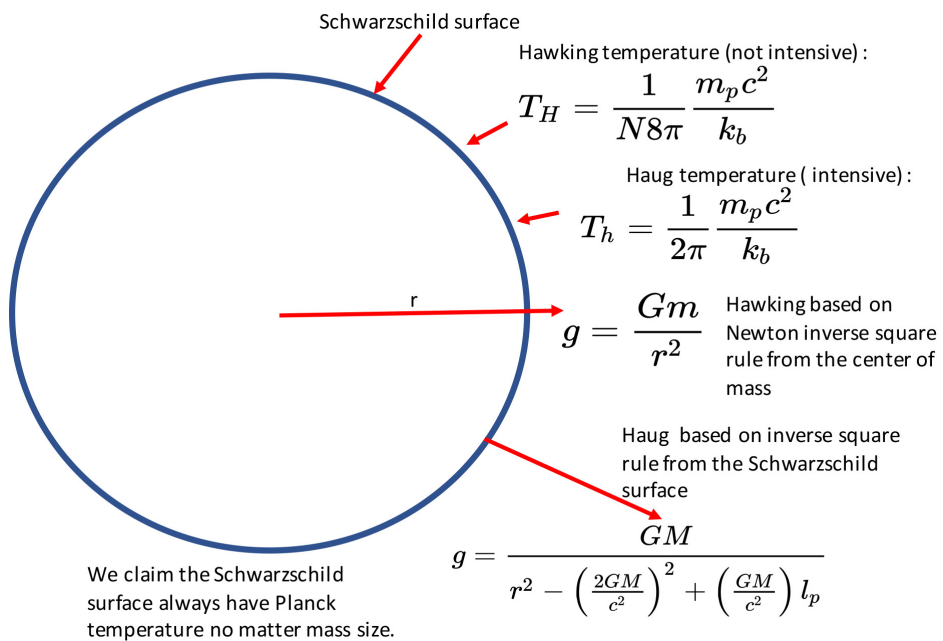

Figure 1. This figure simple illustrates how we assume what is relevant for the gravitational acceleration field is the distance from the Schwarzschild surface, and not from the very center of the gravity object. This will only have implications (predict very different results than standard theory) when very close to the Schwarzschild surface. The arrow from the middle does not indicate that the standard gravitational formula holds inside the Schwarzschild radius; it simply indicates that the gravitational acceleration field at the Schwarzschild radius in the standard theory is calculated using a radius emanating from the center of the gravitational object

However, it is generally assumed that we must know $G$ in order to calculate any Planck units such as the Planck length and the Planck mass. McCulloch (2016) has also assumed that one needs to know $G$ and therefore we get a circular problem

In the above gravitational derivation, the correct value for the gravitational constant $G$ can only be obtained when it is assumed that the gravitational interaction occurs between whole multiples of the Planck mass, but this last part of the derivation involves some circular reasoning, since the Planck mass is defined using the value for $G$.

We, on the other hand, have shown how the Planck length and the Planck mass, both theoretically and experimentally, can be found easily with no knowledge of Newton's gravitational constant Haug $(2017,2018 \mathrm{~d}$,a). This means that $G$ is a composite constant and that the Planck length can be found independent of $G$. The Hawking temperature can therefore also be rewritten as (known)

$$
\begin{aligned}
T_{H} & =\frac{c^{3}}{8 \pi G M} \frac{\hbar}{k_{b}} \\
T_{H} & =\frac{c^{3}}{8 \pi \frac{l_{p}^{2} c^{3}}{\hbar} N m_{p}} \frac{\hbar}{k_{b}} \\
T_{H} & =\frac{c^{3}}{8 \pi \frac{l_{p}^{2} c^{3}}{\hbar} N \frac{\hbar}{l_{p}} \frac{1}{c}} \frac{\hbar}{k_{b}}=\frac{1}{N 8 \pi} \frac{\hbar c}{l_{p} k_{b}}=\frac{1}{N 8 \pi} \frac{m_{p} c^{2}}{k_{b}}
\end{aligned}
$$

where $N$ is the number of Planck masses, $m_{p}$, in the black hole (gravity object). Only when $N=1$, that is for one Planck mass the Hawking temperature gives the "same" prediction as our theory. Haug has recently shown that all well-known gravity predictions and observations can be done without any knowledge of the Newtonian gravitational constant $G$. It is the Planck length that is important for gravity, and it can be found independent of any knowledge of both $G$ and $\hbar$. 
Also, the Schwarzschild radius embedded contains the Planck length, and it actually requires less information to find the Schwarzschild radius than $G$ itself; the standard Schwarzschild radius formula is not needed to find the Schwarzschild radius itself, see section 6. Our theory for a so-called black hole temperature (Hawking temperature) differs from Hawking in that we think the inverse square law of gravity for a gravity mass packed inside a Schwarzschild radius should hold from the Schwarzschild surface, not from the very center of the object. The standard gravity method implies that mass (energy) can be infinitely densely packed, something we strongly doubt, there are also several other issues and problems with the standard approach that we will discuss in the next sections.

\section{Possible Implications for Quasars}

As quasars are considered black holes (at least at their core), our modified Schwarzschild radius (Hawking) radiation should be valid for them as well. Our new model predicts that the quasar radiation is much higher than expected at the surface of the Schwarzschild object (quasar). This also means that the life expectancy of quasars would be much shorter than expected from modern cosmology. Particles with mass around the quasar will be moving towards the quasar due to gravity and will reflect radiation out from the quasar back to the quasar and this will also increase the life expectancy, although that would still be much shorter than expected from standard theory. Recently, astronomers LaMessa (2015) and Sheng et al. (2017) have observed quasars vanishing (or at least changing dramatically) within a 10-year period, where previous theories predicted that this would take at least ten thousand years

Although quasars turn off, transitioning into mere galaxies, the process should take 10,000 years or more. This quasar appeared to have shut down in less than 10 years - a cosmic eyeblink. - Hall (2018)

Our theory also indicates that the quasars must be extremely bright objects, which they are indeed.

\section{The Black Hole Interpretation Crisis?}

Crothers (2012) has been very critical towards the modern physics interpretation of so-called black holes. We think he could be right in some of his criticism. However, based on recent progress in theoretical and applied physics, we also think that the Schwarzschild radius represents something very important related to gravity Haug (2018b). The Schwarzschild radius is the reduced Compton frequency of the gravity object per Planck second times the Planck length. Both the reduced Compton frequency per Planck second and the Planck length can be found independent of GR and also independent of any knowledge of big $G$, see Haug (2018a). We claim that even particles with less mass than the Planck mass have a Schwarzschild radius, but the Schwarzschild radius is then probabilistic, see Haug (2018e, 2019). We suggest that black holes should be understood from a totally new perspective, namely mathematical atomism, which is linked directly to a new and revolutionary understanding of the Planck scale.

A series of modern physics predictions around AGN (quasars/black holes) seem to be totally different than what is observed, such as expected time-dilation in high Z quasars Hawkins (2010); Hawkings (2001), as well as how fast quasars can vanish or change their observable characteristics. In addition, the fact that the Hawking temperature is not intensive should encourage us to question the incompleteness of the fundamental principles in existing theories and look more closely at criticisms as well as potential alternatives; it is useful to take a closer look at the foundation.

\section{The Planck Mass Particle and Micro Black Holes an Alternative Interpretation}

It was Max Planck $(1899,1906)$ who first introduced the Planck length, the Planck mass, the Planck time, and the Planck energy (temperature). He assumed that the most important universal fundamental constants were the speed of light, the Newton gravitational constant, and the Planck constant, and from dimensional analysis he then derived the Planck mass to be $m_{p}=\sqrt{\frac{c \hbar}{G}}$. He developed similar equations for the other Planck units. Even if Planck thought this was an essential mass, he was not clear on exactly how it played a role.

While working at the Rutherford Laboratory, Lloyd Motz (1962, 1966, 1971) suggested that there could be a very fundamental particle with a mass equal to the Planck mass. This particle Motz named the uniton. ${ }^{1}$ Motz suggested that the uniton (Planck mass particle) could be the most fundamental of all particles and that all other particles were initially made of Planck mass particles. Motz acknowledged that his Planck mass (approximately $2.18 \times 10^{-8} \mathrm{~kg}$ ) particle had far too much mass compared to any observed subatomic masses (and even atomic masses). To get around this challenge, he claiming the unitons had radiated most of their energy away:

According to this point of view, electrons and nucleons are the lowest bound states of two or more unitons that have collapsed down to the appropriate dimensions gravitationally and radiated away most of their energy in the process. - Lloyd Motz

\footnotetext{
${ }^{1}$ See also Markov (2014), who introduces a similar particle that he calls maximons.
} 
Others have suggested that there were plenty of Planck mass type particles around just after the Big Bang, see De (2001), but that most of the mass of these super-heavy particles has radiated away. Further Motz and also Hawking (1971) and others have speculated on that Planck mass "particles" are micro black holes, see also Motz and Epstein (1979); Obermair (2013). This because when a particle has a Compton wavelength equal to the Planck length then its escape velocity is $c$ when the reduced Compton wavelength are used as the radius. The Planck mass particle is the smallest particle that is consistent with the assumptions behind black holes. Still no micro black holes have ever been observed. There were also serious discussions when starting up the Large Hadron Collider (LHC) could initiate a catastrophic black hole, see Steven and Michelangelo (2008); Koch, Bleicher, and Stoecker (2009) for discussion on this.

Planck mass particles have even been suggested as candidates for cosmological dark matter, Macgibbon (1987); Dokuchaev and Eroshenko (2014). Others, like Crothers and Dunning-Davies (2006), have strongly criticized the black hole interpretation of the Planck particle and have questioned its existence. Yet, the existence of Planck mass-size particles remains an unsolved mystery, which is linked to the hierarchy problem. However, the current interpretations of the Planck mass and Planck type particles do not seem to provide the entire story and there are actually fresh and alternative ways of looking at matter and energy. Here we will assume there ultimately is only one fundamental particle and this particle makes up all other particles, as well as energy. We will assume that this particle has the following properties:

1. Indivisible particle with a diameter of $l_{p}$, and when at "rest" the mass is equal to half that of the Planck mass.

2. This indivisible particle is always traveling at the speed of light, $c$, as measured with Einstein-Poincaré synchronized clocks, except when colliding with other indivisible particles.

3. This particle travels in the void ("empty space"). This is necessary, so the particle has something to travel in.

In other words, our indivisible particle has half the mass of the uniton particle suggested by Motz in 1962 (Planck masssize particle). In 1979, Motz and Epstein (1979) suggested there likely existed a fundamental particle with half the Planck mass, that is exactly the same mass as in the particle suggested here. Still, they did not have a good explanation for why this particle was so much larger than all existing subatomic particles, what this "particle" truly was, and if it was indivisible or not. Instead of assuming that most of this super-heavy particle mass has radiated away, we will suggest that all mass (and energy) of the indivisible particle (Planck particle) still exist inside each known subatomic particle and even inside energy.

We suggest that collisions between two indivisible particles constitute the only pure mass, and that its mass is the Planck mass if measured over one Planck second. This is the mass-gap and it is observational time dependent due to the fact that it only lasts for one Planck second. If one measures it over one second, the mass is only $m_{p} t_{p} \approx 10^{-51} \mathrm{~kg} \cdot \mathrm{s}$. We claim all other elementary masses switch between internal energy and mass at their Compton frequency. For example, an electron will then have a mass of

$$
\frac{c}{\bar{\lambda}_{e}} m_{p} t_{p}=\frac{c}{\bar{\lambda}_{e}} \frac{\hbar}{l_{p}} \frac{1}{c} \frac{l_{p}}{c}=\frac{\hbar}{\bar{\lambda}} \frac{1}{c}=m_{e} \approx 9.1 \times 10^{-31} \mathrm{~kg}
$$

This possibly has a parallel to Schrödinger (1930)'s idea of a trembling motion (Zitterbewegung) of the electron, where he assume the electron trembled $\frac{2 m_{e} c^{2}}{h}=\frac{c}{2 \lambda_{e}} \approx 1.6 \times 10^{21}$ times per second. In our model, the electron moves in and out of the Planck mass state $\frac{c}{\bar{\lambda}_{e}}$ times per second. But each Planck mass state only lasts for Planck second.

The mass of elementary particles, such as the electron, will be observational time dependent in this model, but this will not be noticeable as long as the observation time is considerably longer than the Compton time interval of the particle (which explains why this has not been observed before). However, recent research in relation to Compton time and mass indicates we could be on the right track, see Lan, Kuan, Estey, English, Brown, Hohensee, and Müller (2013); Dolce and Perali (2015).

The life expectancy of a so-called micro black hole would then only be one Planck second. However, the micro black hole has, in this view, little to do with the modern interpretation of black holes. The micro black hole in this model is not a hole at all, it is the collision point between indivisible (light) particles, that again make up all energy and matter. Their collision point is pure mass, and when they are free moving, they are energy. And all observed particles are internally switching between pure energy and the Planck mass at the Compton frequency. Still, this entity of two colliding indivisible particles have much in common with the properties of a black hole. Nothing can escape it without moving at the speed of light. This is simply due to the Planck mass particle being a collision point of the building blocks of photons, so it dissolves into what it was originally made of, namely light particles. The Planck mass particle here is a photon-photon collision, 
and it is also assumed in modern physics that photon-photon collision can result in matter (mass) (Pike, Mackenroth, Hill, \& Rose, 2014). In this view, all matter consists of Planck mass collisions that last for one Planck second. But again, it is only over a time window of one Planck second that one can observe this mass to be the Planck mass. Other masses smaller than the Planck mass, such as the electron, must then be probabilistic mass when observed at time scales below the reduced Compton time. This is covered in more detail in Haug (2014, 2016b, 2018e).

The Schwarzschild radius is normally given as $r_{s}=\frac{2 G M}{c^{2}}$. It has recently been shown by Haug (2019) that the Schwarzschild radius can be extracted from gravity observations with no knowledge of Newton's gravitational constant, the mass size, or even the speed of light. The Schwarzschild radius is given by the following formula

$$
r_{s}=\frac{R R_{h}\left(\lambda_{h}^{2}-\lambda^{2}\right)}{\lambda_{h}^{2} R_{h}-\lambda^{2} R}
$$

where $\lambda$ and $\lambda_{h}$ are respectively the observed wavelength of a light beam at radius $R$ and $R_{h}$, (where $R_{h}>R$ ). It can alternatively be written in the frequency form; $r_{s}=\frac{R R_{h}\left(f^{2}-f_{h}^{2}\right)}{f^{2} R_{h}-f_{h}^{2} R}$. That is all we need to find the Schwarzschild radius is a light beam, for example sent down from a tower and to measure the redshift of the beam, similar to the Pound and Rebka Jr. (1959) experiment. Only when we want to find the weight of the gravity mass do we need to know $G$. We claim that the Schwarzschild radius is the reduced Compton frequency of matter over the shortest possible time interval multiplied by the Planck length and that it plays a major role for all gravity phenomena. Further, from the Schwarzschild radius basically all standard gravity phenomena can be predicted as recently shown by Haug (2018b, 2019). This means a series of major gravity phenomena only need a few observations of light to be predicted accurately. One should ask how can it be a series of gravity phenomena only can be predicted from only observations of a light beam. In our view, this strongly indicates that there is a close connection between electromagnetism and gravity; even if it is not fully understood yet, this may bring us one step closer to making that connection more clearly. Many physicists have worked on a trying to unify quantum mechanics and gravity for over 100 years; perhaps the answer lies in having a fresh look at the Schwarzschild radius and the Planck mass particle.

\section{Conclusion}

Crothers and Robitaille have recently pointed out that the Hawking temperature is not intensive and noted that this is in conflict with thermodynamics. Thus, we may ask, "Is the Hawking temperature incomplete or flawed in some way?" Haug has suggested a modified Newton gravitational acceleration field that gives the same gravitational acceleration in weak gravitational fields as predicted by Newton and as observed, but that gives very different gravitational acceleration linked to the Planck scale at the Schwarzschild radius in very strong gravitational fields. Using this modified gravitational acceleration field, we obtain an intensive temperature at the Schwarzschild radius. We think the intensive crisis contains significant information and that alternative theories about gravity should be examined more closely. We have also shown that the Schwarzschild radius can be extracted from gravity observations with no knowledge of Newton's gravitational constant, the speed of light, or the mass size of the object. The Schwarzschild radius is, in our view, the reduced Compton frequency of the gravity object over the shortest possible time interval multiplied by the shortest possible length, and this is what is important for gravity. In this framework, many interpretations around black holes could have an alternative explanation, and further investigation is warranted.

\section{Acknowledgements}

Thanks to Victoria Terces for helping me edit this manuscript and thanks to Greg Jenkins and an anonymous referee for useful comments.

\section{References}

Cavendish, H. (1798). Experiments to determine the density of the Earth. Philosophical Transactions of the Royal Society of London, (88), 469-526.

Crothers, S. J. (2012). Proof the the Black Hole Has No Basis in General Relativity or Newtonian Gravitation and Therefore Does Not Exist. Retrieved from http://vixra.org/pdf/1212.0010v1.pdf.

Crothers, S. J., \& Dunning-Davies, J. (2006). Planck particles and quantum gravity. Progress in Physics, 3(2006), 70.

Crothers, S., \& Robitaille, P. M. (2018). The Unruh Effect: Insight from the Laws of Thermodynamics. Bulletin of the American Physical Society. Retrieved from http://vixra.org/pdf/1811.0157v1.pdf

Crothers, S. J., \& Robitaille, P. M. (2019). Intensive and Extensive Properties: Thermodynamic Balance. Physics Essays, 
32.

De, S. S. (2001). Quantum creation of highly massive particles in the very early universe. International Journal of Theoretical Physics, 40(11), 2067-2084.

Dokuchaev, V. I., \& Eroshenko, Y. N. (2014). Black hole atom as a dark matter particle candidate. Advances in High Energy Physics, 2014.

Dolce, D., \& Perali, A. (2015). On the Compton clock and the undulatory nature of particle mass in graphene systems. The European Physical Journal Plus, 130(3), 41.

Hall, S. (2018). Galactic Beacons Get Snuffed Out in a Cosmic Eyeblink. Quanta Magazine.

Haug, E. G. (2014). Unified Revolution: New Fundamental Physics. Oslo: E.G.H. Publishing.

Haug, E. G. (2016a). The gravitational constant and the Planck units. A simplification of the quantum realm. Physics Essays, 29(4), 558-561.

Haug, E. G. (2016b). The Planck mass particle finally discovered! The true God particle! Good bye to the point particle hypothesis. Retrieved from http://vixra.org/abs/1607.0496.

Haug, E. G. (2016c). Planck Quantization of Newton and Einstein Gravitation. International Journal of Astronomy and Astrophysics, 6(2).

Haug, E. G. (2017). Can the Planck length be found independent of big G. Applied Physics Research, 9(6).

Haug, E. G. (2018a). Finding the Planck Length Independent of Newton's Gravitational Constant and the Planck Constant: The Compton Clock Model of Matter. Retrieved from https://www.preprints.org/manuscript/201809.0396/v1, Submitted to journal.

Haug, E. G. (2018b). Gravity without Newton's Gravitational Constant and No Knowledge of Mass Size. Retrieved from https://www.preprints.org/manuscript/201808.0220

Haug, E. G. (2018c). Maximum Velocity for Matter in Relation to the Schwarzschild Radius Predicts Zero Time Dilation for Quasars. Retrieved from https://www.preprints.org/manuscript/201810.0674

Haug, E. G. (2018d). Planck Mass Measured Totally Independent of Big G Utilising McCulloch-Heisenberg Newtonian Equivalent Gravity. Retrieved from https://europepmc.org/abstract/ppr/ppr51310

Haug, E. G. (2018e). Revisiting the Derivation of Heisenberg's Uncertainty Principle: The Collapse of Uncertainty at the Planck Scale. Retrieved from https://www.preprints.org/manuscript/201805.0258

Haug, E. G. (2018g). A Simple Newtonian Quantum Gravity Theory That Predicts the Same Light Bending as GR. And also a new gravitational prediction that can be tested!. Retrieved from www.viXra.org 1802.0169

Haug, E. G. (2019). Extraction of the speed of gravity (light) from gravity observations only. International Journal of Astronomy and Astrophysics, Accepted.

Hawking, S. (1971). Gravitationally collapsed objects of very low mass. Monthly Notices of the Royal Astronomical Society, 152(1), 75-78.

Hawking, S. W. (1974). Black hole explosions?. Nature, 248(5443), 30.

Hawking, S. (1976). Black Holes and Thermodynamics. Physical Review D, 13(191).

Hawkins, M. R. S. (2001). Time dilation and quasar variability. The Astrophysical Journal Letters, 553(2), 97-100.

Hawkins, M. R. S. (2010). On time dilation in quasar light curves. Monthly Notices of the Royal Astronomical Society, 405(3), 1940-1946.

Hees, A., Bailey, Q., Bourgoin, A., Bars, P. L., Guerlin, C., \& Poncin-Lafitte, L. (2016). Tests of Lorentz symmetry in the gravitational sector. Universe, 2(4), 30. 
Hochberg, D., \& Prez-Mercader, J. (1997). Liquid model analogue for black hole thermodynamics. Physical Review D, $55(8), 4880$.

Koch, B., Bleicher, M., \& Stoecker, H. (2009). Exclusion of black hole disaster scenarios at the LHC. Physics Letters B, 672(1), 71-76.

LaMassa, S. M., Cales, S., Moran, E. C., Myers, A. D., Richards, G. T., Eracleous, M., ... \& Urry, C. (2014). The Discovery of the First" Changing Look" Quasar: New Insights into the Physics \& Phenomenology of AGN. arXiv preprint arXiv:1412.2136.

Lan, S. Y., Kuan, P. C., Estey, B., English, D., Brown, J. M., Hohensee, M. A., \& Mueller, H. (2013). A clock directly linking time to a particle's mass. Science, 339(6119), 554-557.

Landsberg, P. Y. (1961). Thermodynamics. New York: Interscience Publishers.

Reyes, C. M., Ossandon, S., \& Reyes, C. (2015). Higher-order Lorentz-invariance violation, quantum gravity and finetuning. Physics Letters B, 746, 190-193.

MacGibbon, J. H. (1987). Can Planck-mass relics of evaporating black holes close the Universe?. Nature, 329(6137), 308.

Markov, M. A. (1967). Elementary particles of maximally large masses (Quarks and Maximons). Sov. Phys. JETP, 24, 584-592.

McCulloch, M. E. (2014). Gravity from the uncertainty principle. Astrophysics and Space Science, 349(2).

McCulloch, M. E. (2016). Quantised inertia from relativity and the uncertainty principle. EPL (Europhysics Letters), $115(6), 69001$

Michell, J. (1784). On the Means of Discovering the Distance, Magnitude etc. of the Fixed Stars, in Consequence of the Diminution of the Velocity of Their Light, in Case Such a Diminution Should be Found to Take Place in any of Them, and Such Other Data Should be Procured from Observations. Philosophical Transactions of the Royal Society, 74.

Motz, L. (1962). Gauge Invariance and the Structure of Charged Particles. Il Nuovo Cimento, 26(4).

Motz, L. (1970). A gravitational theory of the $\mu$-meson and leptons in general. Il Nuovo Cimento A (1965-1970), 65(2), 326-332.

Motz, L. (1971). 'The Quantization of Mass. Rutherford Observatory, Columbia University.

Motz, L., \& Epstein, J. (1979). The Gravitational Charge $1 / 2 \sqrt{\hbar c}$ as a Unifying Principle in Physics. Il Nuovo Cimento, 51(1).

Newton, I. (1686). Philosophiae Naturalis Principia Mathematica. London.

Obermair, G. M. (2013). Primordial Planck mass black holes (PPMBHs) as candidates for dark matter?. Journal of Physics, Conference Series, 442.

Pike, O. J., Mackenroth, F., Hill, E. G., \& Rose, S. J. (2014). A photon-photon collider in a vacuum hohlraum. Nature Photonics, 8(6), 434.

Planck, M. (1899). Natuerliche Masseinheiten. Der Königlich Preussischen Akademie Der Wissenschaften.

Planck, M. (1906). Vorlesungen über die Theorie der Wärmestrahlung. Leipzig: J.A. Barth. see also the English translation "The Theory of Radiation" (1959) Dover.

Pound, R. V., \& Rebka Jr, G. A. (1959). Gravitational red-shift in nuclear resonance. Physical Review Letters, 3(9), 439-441.

Robitaille, P. M. (2018). Hawking Radiation: A Violation of the Zeroth Law of Thermodynamics. http://vixra.org/pdf/1803. 0264v1.pdf. 
Schrödinger, E. (1930). Über die kräftefreie Bewegung in der relativistischen Quantenmechanik. Sitzungsberichte der Preuischen Akademie der Wissenschaften. Physikalisch-mathematische Klasse.

Giddings, S. B., \& Mangano, M. L. (2008). Comments on claimed risk from metastable black holes. arXiv preprint arXiv:0808.4087.

Sheng, Z., Wang, T., Jiang, N., Yang, C., Yan, L., Dou, L., \& Peng, B. (2017). Mid-infrared Variability of Changing-look AGNs. The Astrophysical Journal Letters, 846(1), L7.

\section{Copyrights}

Copyright for this article is retained by the author(s), with first publication rights granted to the journal.

This is an open-access article distributed under the terms and conditions of the Creative Commons Attribution license (http://creativecommons.org/licenses/by/4.0/). 